\title{
Trombosis carotidea secundaria a traumatismo oral
}

Drs. Carlos Brousse E., Juan C. Casteran V., Maria E. Fermández

\begin{abstract}
Carotid thrombosis secondary to oral trauma
A case of carotid thrumbosis due to intraoral trauna in a 6 years old girt is reported.

Literature retiew, clinical picture and necropsy of this case is presented. Specikt interest in the treatrinent of cerebral swelling is fiocused.
\end{abstract}

El traumatismo de la carótida interna por via intraoral es un accidente muy infrecuente en la infancia. Hasta la fecha se han descrito 17 casos en la literatura mundial, siendo el primero presentado por Caldwell en el año 1936.

Habitualmente la injuria es producida por un objeto punzante que el niño se ha llevado a la boca; otras veces es el producto de una caida sobre el objeto punzante que penetra en la boca y daña la región tonsilar. El traumatismo no necesariamente debe ser penetrante.

Es bien conocida la proximidad de la carótida interna al lecho tonsilar. Se describen desgarros de la íntima de este vaso sin penetración de la pared (Verneuil y Greco, Caldwell y Hadden). Braudo postula que esta lesión sirve de nido para la formación de trombos, que progresan hasta la obstrucción total del lumen. Bickerstaff comunicó un aneurisma disecante de la pared de la carótida interna detrás de la fơsa tonsilar y distalmente en la primera porción de la arteria cerebral media, con intarto cerebral en el territorio de la arteria cerebral media.

Shannon y cols. describieron un desgarro de la intima de la carótida interna detrás del paladar blando, en el sitio del trombo que se extendió distalmente en el territorio de las arterias cerebral media y anterior. El objeto penetrante habria pasado muy próximo a la carótida interna. Se describió intenso edema y reblandecimiento cerebral. La angiografía confirmó el sitio de la trombosis en el lugar del traumatismo con propagación distal y proximal del trombo y embolización distal. Pittner postula que un traumatismo en la carótida interna puede producir un desgarro de la íntima con formación de trombo oclusivo. Como muchos individuos, especialmente los niños, pueden tolerar oclusión carotidea unilateral sin sintomas neurológicos, suele no haber déficit inmediato. Los signos neurológicos son el resultado de la embolización asterial, habitualmente de la arteria cerebral media. Esto explica que la gran mayoria 
de los pacientes presentan un período de latencia entre el accidente $y$ la aparición de manifestaciones psicomotoras (0-24 horas).

Inicialmente el tratamiento fue de soporte y sintomático. Con posterioridad se intentó la simpatectomía por bloqueo del ganglio estrellado.

La exploración carotidea se demostró inútil, incluso la endarterectomía, con aparente recuperación de flujo, se complicó posteriormente con trombosis. Los anticoagulantes se usaron en un solo caso, con efectos poco claros.

El último caso fue descrito por Woodhurst en una niña de 2 años que sobrevivió a un gran intarto del hemisferio cerebral derecho con edema cere. bral y hemia transtentorial, en quien se usó terapia agresiva contra el edema cerebral.

El pronóstico puede variar desde alteraciones neurológicas leves y transitorias hasta la muerte. La mortalidad en los pacientes reportados asciende a un $30 \%$. La revisión de los casos publicados sugiere que la muerte se debe a hemia transtentorial secundaria a edema e infarto cerebral.

\section{Caso Clinico}

Paciente de sexo femenino, de 6 años de edad, que mientras jugaba sobre su cama se hirió con un lápiz en la región paratonsilar izquierda del velo del paladar. El traumatismo le provocó intenso dolor, presentando repercusión sistémica, casacterizada por apnea y palidez intensa. Permaneció asintomática durante las seis horas que siguieron al traumatismo, al cabo de las cuales se quejó de cefalea intensa, pero logró conciliar el sueño. Seis horas más tarde despertó gritando, desconectada y aparentemente presentó una crisis convulsiva generalizada, con desviación ocular, que cedió espontaneamente. Al tratar de incorporarla, su madre notó pérdida de fuerzas en las extremidades derechas.

Ingresó a nuestro Servicio 18 horas después de producido el accidente con compromiso de conciencia y tendencia al sopor; al estimularla, despertaba y obedecía algunas órdenes verbales; había afasia motora, hemiparesia faciobraquiocrural derecha, disminución del tono, pero con conservación de retlejos protundos; respuesta plantar extensora en el lado derecho; abolición de retlejos cutáneos abdominales derechos. La exploración de pares craneanos reveló paresia de la nuirada conjugada hacia la derecha, paresia facial derecha de tipo central y hemianopsia homónima derecha.
Sin signos de hipertensión endocraneana. Fondo de ojos y líquido cefalorraquídeo nomales. En la región faríngea se comprobo una pequeña herida punzante en el velo del paladar, en la región paratonsilar izquierda.

Los hallazgos descritos corresponden a compromiso del hemisferio izquierdo, en el territorio de la arteria cerebral media y fueron interpretados como un proceso obstructivo secundario a arteritis.

Se formuló el diagnóstico de hemiplejía infantil aguda y se tomaron medidas contra el edema cerebral consistentes en restriccion hídrica y corticoides (Solucortef $40 \mathrm{mg} / \mathrm{kg}$ cada 6 horas E.V.).

La radiografía de cráneo fue nomal. No fue posible realizar electroencefalograma $y$ angiografia carotidea por no disponer de estos recursos.

En el tercer día de hospitalización aumentó su compromiso de conciencia, con regresión de su hemiparesia, lo que no era concordante con el diagnóstico inicial de hemiplejía infantil aguda; se encontraba en coma grado III a IV, con respiración profunda a ratos periódica; tenía movimientos espontáneos de las cuatro extremidades, con tendencia a la pronación de las extremidades superiores, hipotonía y exaltación de los reflejos profundos, sensibilidad superficial abolida y profunda francamente disminuida, anisocoria, midriasis más acentuada a la izquierda y escasa respuesta fotomotora. En el fondo de ojos la papila era normal y no había latidos venosos.

Glicemia, uremia, transaminasas, protrombina, ionograma y nuevo examen de líquido cefalorraquídeo eran nomales. Hemograma: $\mathrm{H}$ to $45 \%$; leucocitos $13.400 \times \mathrm{mm}^{3}$, sin al teraciones morfológicas (segmentados 72; linfocitos 20; monocitos 8). Plaquetas normales. VHS: $17 \mathrm{~mm}$ en la 1. a hora. Cultivos de L.C.R. (-).

Al tratamiento prescrito se agregó Manitol 0,75 g/kg cada 6 horas E.V.; Fenobarbital $10 \mathrm{mg} / \mathrm{kg} / \mathrm{día}$ I.M. y medidas de prevención de úlcera de stress. En la madrugada del día siguiente presentó paro respiratorio, indicándose respiración mecánica. Se aumentó la dosis de Fenobarbital a $30 \mathrm{mg}$ kgidia y se suspendió el Manitol. Se agregó Penicilina sódica $100.000 \mathrm{U} / \mathrm{kg} /$ dia; Quemicetina 50 $\mathrm{mg} / \mathrm{kg} /$ dia y Amikasina $15 \mathrm{mg} / \mathrm{kg} /$ día, en consideración al alto riesgo de infección derivada de la ventilación mecánica.

En los diás sucesivos continuó en coma, sin respiración espontánea, con midriasis paralítica bilateral, hipotonía generalizada, arreflexia y evi- 
dente tendencia al shock, el que se manejó sucesivamente con Isuprel ${ }^{(\mathbf{k})}$ y Dopamina en dosis de hasta $9 \mu \mathrm{g} / \mathrm{kg} / \mathrm{min}$, sin respuesta satisfactoria. Falleció ocho dias después de ingresar.

\section{AVATOMIA PATOLOGICA}

Cerca de la amigdala izquierda se encontró una zona de infiltración pardusca que se prolongaba hasta la base del cráneo. La disección de la arteria carótida interna izquierda demostró una trombosis masiva que se extendia a todo el territorio de la arteria cerebral nedia. El cerebro estaba tumefacto, con aumento difuso de volumen, mís intenso en el lado izquierdo, aplanamiento de las circunvoluciones y reblandecimiento especialmente en la región occipital. Tronco encefálico edematoso, hacia hernia a través del agujero occipital. Infarto masivo de hemisferio cerebral izquierdo.

\section{DISCUSION}

Este caso revela las fatiles consecuencias que puede acarrear un tramatismo intraoral considerado inicialmente inccente.

Las características del accidente, el perísdo asintomático, la aparición posterior de signos neurológicos y complicaciones debidas al intenso edema cerebral son el todo similares a lo descrito en la literatura.

Desgraciadamente no pudimos contar con angiografia carotidea que nos confirmara la hipotes is clínica en este caso.

La presencia de shock, probablemente neurogénico, y las alteraciones cardiortespiratorias nos hicieron sospechar compromiso troncoencefálico debido a infarto y edema cerebral, el que fue confirmado posterionmente en la necropsia.
Concordanos con la literatura en que el manejo de estos pacientes debe ser enfocado al tratamiento oportuno del edema cerebral, para evitar el enclavarniento, causa final de la muerte.

Creemos que estos accidentes podrian ser prevenidos con medidas de sentido común. En lo posible debe evitarse el acceso de los niños a objetos punzantes y enseñarles a no introducirlos en la boca. Ocurrido el accidente, debe mantenerse estrecha vigilancia por un lapso de 2 a 3 días, tratando de pesquisar manifestaciones neurológicas, de manera de hacer diagnóstico y tratamiento oportumos.

\section{RESUMEN}

Se presenta el caso de una niña de 6 años con trombosis de carótida intema secundaria a traumatismo intraoral. Se revisan la literatura, el cuadro clínico y la necropsia del caso. Se hace hincapié en el tratamiento del edema cerebral.

\section{REFERENCIAS}

I Bickerstaff, E R. Aetiolokgy of acunte hemiplegia in childhond. Br. Med. J., 2: 62-87, 1964 .

2 Braudo, $M$. Thrumbosis of intenal carotid artery in childhood aliter iṇ̂iuries in region ol solt pulate. Br. Med. J.. t: 665-66?. 1456.

3 Fairbum, B. Thrumbosis of interual carotid artery after soft palate injury. Br. Med. J., 2: 750, 1457.

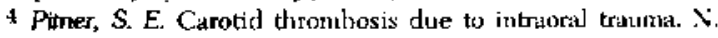
Engl. J. Med., 274: 764-767, 1966i

5 Shannon, E.; Cohn, D.; Streifler, M., and Fapoport. Y, Penetrating injuries of the partphtaryingeal space. Arch. Otolaryngol. 96: 256-259, 1972 .

6 Tomealba, G; Tagle, $P$. Trombasis carotidea en el lactante. Hev. Chil. Ped., Vol. 45, pag. 63, 1974.

7 Woodhurs, W. B.; Robertson, W. D.; Thompson, G. B. Carotid injury due to intraoral tranma: Case report and review of the literature. Nenrosurg, Vol. 6, N. 5, 559-563, 1980. 